\title{
Human Face Pose Estimation based on Feature Extraction Points
}

\author{
Guneet Bhullar \\ Research scholar, \\ Department of ECE \\ SBSSTC, Moga Road, \\ Ferozepur, Punjab, India
}

\author{
Vikram Mutneja \\ Assist. Professor, \\ Department of ECE \\ SBSSTC, Moga Road, \\ Ferozepur, Punjab, India
}

\begin{abstract}
The process of Face Recognition comprises of Face Detection, feature extraction and verification or identification. The extraction and identification are stages in the FR process. Many face recognition algorithms have been developed. This has resulted in development of manifold robust techniques such as background removal, illumination normalization and others which support the algorithm to withstand the undesirable effects and improve the success rate. This paper provides a survey and method for face pose estimation. This method is based on feature extraction points of two different face poses and then matched points between these two face poses will give the results. This method is one of the simplest methods for low resolution images.
\end{abstract}

\section{General Terms}

Face Recognition, Face pose, Feature Points.

\section{Keywords}

Feature extraction, low resolution, roll, pitch, yaw, orientation.

\section{INTRODUCTION}

In the last two decades, face pose estimation becomes active field of research. During this period, the main aim of researchers has become innovative. Previous works has been able to identify some predefined poses. In the recent years, the problem of human interface has been very important in research fields such as computer vision, human identification, gaming, other medical services etc. therefore many researcher works in this field in order to investigate and recognize human interface issues. Among these entire problems, face recognition system gets great attention.[1] This recognition system includes face detection and identification and face pose estimation. Generally, this system relies on feature based methods. Nowadays, face pose estimation achieves precision and less feature points which can enhance the estimation speed. Various techniques can be categorized into three parts: (1) appearance-based approaches. (2) model-based approaches. (3) Video streams based approaches. In appearance based approach, evaluate the face pose through machine learning. In model based approaches, it evaluates face poses through the relationship between facial feature points and face rotation.

Face recognition (FR) is a challenging issue owing to its complexity and costly usage in applications in fields such as forensics, vigilance, Law enforcement, user access, human computer interaction and for various other security purposes. Many commercial systems thus use face recognition. The process of Face Recognition consists of Face Detection, feature extraction and verification or identification. The extraction and identification are stages in the FR process.
Many face recognition algorithms have been developed. However due to position, pose, noise and variations in the input image, the success rate of the algorithms is not deterministic. This has resulted in development of innumerable robust techniques such as background removal, illumination normalization and others which support the algorithm to combat the undesirable effects and improve the success rate.[2] Feature extraction using block based DCT involves dividing the image into blocks of uniform size and isolating the most relevant features of each block. The DC component or low frequency components contain the maximum relevant information useful for face recognition with illumination variation effects, while the high frequency components correspond to details like edges and expressions which are vulnerable to pose and expression variations. In face pose estimation, some feature points are chosen such as nose tip, pupil, and center line of mouth. In these feature point some factor may be considered: i. When the expression of face changes, then the position of pupils changes, but there will be no change in the nose tip. ii. The three points are high brightness in infrared images .iii. Three points are visible only when yaw and pitch have larger angle. The error occurs in face pose estimation when their will be a change in the distance between the camera and face and change in rotation. To remove this error, face normalization is required.[3] Usually, edge information of mouth and nose is higher than the other .the integral value of nose and mouth area is large and area between them is small. Then it can estimate nose tip to determine the center line of mouth, initially mouth area is required only then center line of moth can be estimated.

Some face recognition algorithms identify features by extracting landmarks, or features, from an image of the subject's face. For example, an algorithm may determine the relative position, size, and/or shape of the eyes, nose, cheekbones, and jaw. These features are then used to search for some other images with matching features. Other algorithms normalize the different face images and then compress these face images with respect to face data, only saving the data in the image that is useful for face recognition. An enquiry image is then compared with the face data. One of the earliest successful systems is based on matching techniques of template that are applied to a set of some facial features, providing a sort of compressed face representation.[4][5] Recognition algorithms can be divided into two main approaches, first is geometric, which look at distinguishing features, and second is photometric, which is a statistical approach that refine an image into values and compares the values with templates in order to eliminate variances.[11]

Basically for evaluation of various face poses different techniques are used. But feature extraction method is one of 
the simplest methods.[12] Knowing the face pose of a person provides important indication concerning visual focus of attention [13]. Applications such as video surveillance, intelligent environments and human interaction modelling require face pose estimation from low-resolution face images. But most methods described in the research literature require high-resolution images, often using different views of the face. [14][15] In this paper, problem of estimating face pose from low-resolution single images are evaluated.

\section{RELATED WORK}

Jiaolong yang [1] proposed an approach for face pace estimation. This is based on the combined 2D and 3D histogram of oriented gradients features. In this paper, multilayer perception network is developed for the estimation of the face orientation. The results of this experiment indicate that this method is very effective.

Bing-Fei $\mathbf{W u}[3]$ presented active appearance model. This approach depends on the K-nearest neighbor classifier for face recognition system. The proposed model has various variations and third model also termed as deformable model. Active appearance model is used to evaluate the non-rigid visual objects. These visual objects are described using mean vector and linear combination of set of variation modes.

In this paper Pedro jimenez [3] discussed automatic 3D model. In this work, real time face pose estimation has been performed under some special conditions. This 3D model is combination set of 3D points which is derived from the stereo gray-scale images. This newly proposed model totally depends on the automatic and runtime incremental 3D face modeling. The final result indicates the precise sparse 3D model.

F. Dornaika [4] proposed a sensitive embedding approach. In this work, Sparse Label sensitive Locality Preserving Projections (Sp-LsLPP) has been presented. The result is compared with various other linear and non-linear approaches and confirms that the proposed approach can outperforms.

Deqiang Li [5] in this paper central profile based 3D face pose estimation method has been presented. Out of the other technique has been presented to investigate the nose point .this is detected by nose model matching algorithm. Furthermore, pitch angel estimation has also been presented in this paper. FRGCv2.03D database illustrate the accuracy and precision of the proposed algorithm.

Xiaoni liang [6] presented a technique to estimate the pose of faces in infrared images. This approach extract nose tip, center line of mouth and pupil automatically. These features are used to estimate the pose rotation of pitch. This technique uses less processing time for each image.

Robert Niese [7] proposed real time face estimation using kinect camera. This approach is accurate, fast and robust. Also, iterative closest point algorithm is implemented in this approach which uses depth sensor in order to create user specific model. The results demonstrate that this method is accurate among other approaches.

In this paper Maria Pateraki[8] presented visual estimation of pointed targets. This technique used Dempster-shafer theory. The experimental result proves the effectiveness of method in real world.

Wuming Zhang [9] proposed 3D face recognition system. This approach has been presented through the progressive pose estimation. In this work, random forest is developed in order to deriving 3D models .moreover; 3D morphable model has also been presented which is very effective and less time consuming approach.

Xiangxin Zhu [10] in this paper model for face pose estimation, face detection has been presented. This model is based on the combinations of trees. The result demonstrates that tree structured model has been effective at capturing elastic deformation.

\section{PROPOSED WORK}

The method used in this paper is based on face feature extraction points. In this method, firstly eigen features of both the faces which are in different poses evaluated. The low resolution images can be given as in Fig 3.(a) .
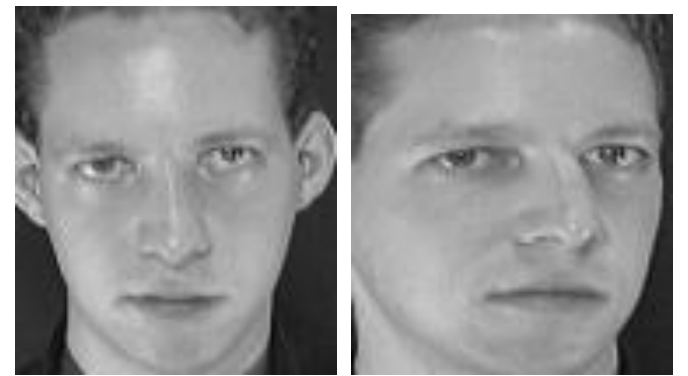

Fig 3.(a) Different poses of face

As shown in the fig, the first face is showing the frontal pose while the second one is showing the face with an orientation. This orientation can be measured with three angles which are roll, pitch and yaw. These orientations are shown in fig 3.(b)

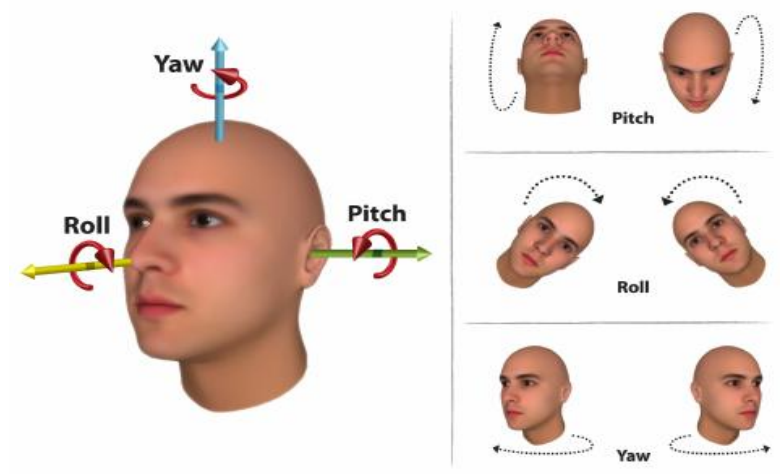

Fig 3.(b) Orientations of face

Now talking about feature points of different face poses, eigen features are detected first. This can be seen in the fig 3.(c).
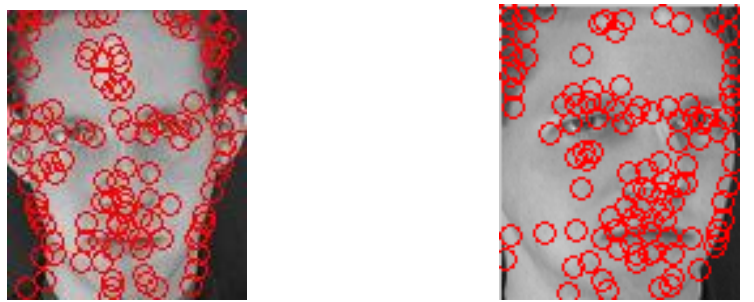

Fig 3.(c) Face feature points of two different pose

After detection of these points, orientation of pose must be evaluated. Now for this evaluation, both the poses should have some relation. This can be done with the help of matching points between two face poses. These matching points will match the features itself. This is shown in fig 3.(d) 


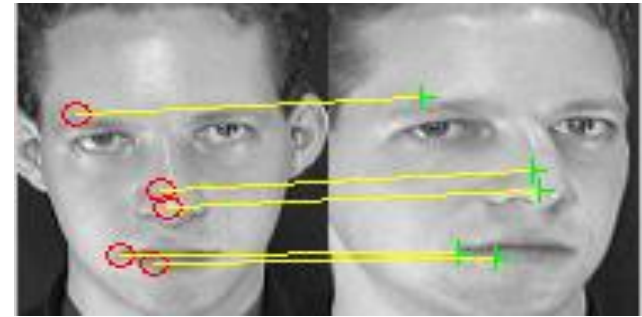

Fig 3.(d) Matched features between two face poses

Now it is easy to measure the orientation from one pose to another. This measurement is done in terms of angles. After decomposition of rotation of face pose, matching points have three angles which are roll, yaw and pitch as discussed earlier. The results can be observed in next section.

\section{RESULTS}

The results of orientation of the human face pose in this paper are evaluated in the form of radians (angle). The results can be given in table

Table 1. Results of variation of different face poses.

\begin{tabular}{|l|l|l|l|l|}
\hline $\begin{array}{l}\text { S. } \\
\text { no }\end{array}$ & $\begin{array}{l}\text { Variation } \\
\text { of poses }\end{array}$ & $\begin{array}{l}\text { Roll (in } \\
\text { radians) }\end{array}$ & $\begin{array}{l}\text { Pitch (in } \\
\text { radians) }\end{array}$ & $\begin{array}{l}\text { Yaw (in } \\
\text { radians) }\end{array}$ \\
\hline 1 & $\begin{array}{l}\text { Front to } \\
\text { left }\end{array}$ & -1.5436 & 0.0245 & 0.4675 \\
\hline 2 & $\begin{array}{l}\text { Front to } \\
\text { right }\end{array}$ & 0.7433 & -0.1345 & -1.3523 \\
\hline 3 & $\begin{array}{l}\text { Front to } \\
\text { upper }\end{array}$ & 0.0678 & 1.2859 & -0.0865 \\
\hline 4 & $\begin{array}{l}\text { Front to } \\
\text { down }\end{array}$ & 0.2648 & -0.8532 & 0.0709 \\
\hline
\end{tabular}

The above shown are the results of variation of different face poses.

\section{CONCLUSION}

The work presented in this paper has main advantage of measurement of orientation of face poses. As there are no bounded limits regarding measurement of roll, pitch and yaw angle. So it is one of the main advantages. But the angle measured is in the form of radians instead in degrees. This method can also be adapted to video sequences for situations such as human interaction modelling, video surveillance and intelligent environments. This can also be improved by removing more and more feature points. There is still much work to be done and large scope of improvement in the estimation as the current techniques have some limitations.

\section{ACKNOWLEDGMENTS}

We are thankful to those who made this learning and research very easy in order for the completion of this paper. This paper contains many important points which are very helpful to find various variations in the human face poses. The dependable topic of this paper is feature extraction points. Once it is clear, it will also helpful in number of applications regarding face recognition.

\section{REFERENCES}

[1] Yang, Jiaolong, Wei Liang, and Yunde Jia. "Face pose estimation with combined 2D and 3D HOG features." Pattern Recognition (ICPR), 2012 21st International Conference on. IEEE, 2012.
[2] Wu, Bing-Fei, Chih-Chung Kao, Cheng-Lung Jen, ChiaRong Chiang, and Po-Hung Lai. "active appearance model algorithm with k-nearest neighbor classifier for face pose estimation."Journal of Marine Science and Technology-Taiwan 22, no. 3 (2014): 285-294.

[3] Jiménez, Pedro, Luis M. Bergasa, Jesús Nuevo, and Pablo F. Alcantarilla. "Face pose estimation with automatic 3D model creation in challenging scenarios." Image and Vision Computing 30, no. 9 (2012): 589-602.

[4] Dornaika, F., C. Chahla, F. Khattar, F. Abdallah, and H. Snoussi. "Discriminant sparse label-sensitive embedding: Application to image-based face pose estimation." Engineering Applications of Artificial Intelligence 50 (2016): 168-176.

[5] Li, Deqiang, and Witold Pedrycz. "A central profilebased 3D face pose estimation." Pattern Recognition 47, no. 2 (2014): 525-534.

[6] Liang, Xiaoni, and Weiqing Tong. "Face pose estimation using near-infrared images." In Communication Systems and Network Technologies (CSNT), 2012 International Conference on, pp. 216-220. IEEE, 2012.

[7] Niese, Robert, Philipp Werner, and Ayoub Al-Hamadi. "Accurate, fast and robust realtime face pose estimation using kinect camera." In Systems, Man, and Cybernetics (SMC), 2013 IEEE International Conference on, pp. 487490. IEEE, 2013.

[8] Pateraki, Maria, Haris Baltzakis, and Panos Trahanias. "Visual estimation of pointed targets for robot guidance via fusion of face pose and hand orientation." Computer Vision and Image Understanding 120 (2014): 1-13.

[9] Zhang, Wuming, Di Huang, Dimitris Samaras, JeanMarie Morvan, Yunhong Wang, and Liming Chen. "3D assisted face recognition via progressive pose estimation." In Image Processing (ICIP), 2014 IEEE International Conference on, pp. 728-732. IEEE, 2014.

[10] Zhu, Xiangxin and Deva Ramanan "Face detection, pose estimation, and landmark localization in the wild." In Computer Vision and Pattern Recognition (CVPR), 2012 IEEE Conference on, pp. 2879-2886. IEEE, 2012.

[11] Nicolas Gourier, Jerome Maisonnasse, Daniela Hall, James L. Crowley "Head Pose Estimation on Low Resolution Images" 1999.

[12] K Schwerdt, J. Crowley, " Robust face Tracking using Color," International Conference on Automatic face and Gesture Recognition pp. 90-95, 2000.

[13] R. Stiefelhagen, "Estimating Head Pose with Neural Networks- Results on the Pointing04 ICPR Workshop Evaluation Data," Pointing 2004, ICPR, Visual Observations of Deictic Gestures, Cambridge, UK.

[14] N. Gourier, D. Hall, and J.L Crowley. "Estimating face orientation from robust detection of salient facial features ".Observation of Deictic Gestures at POINTING04, 2004.

[15] Satyanarayanan M.(2001) Pervasive Computing Vision and Challenges IEEE Personal Communications 8, pp. $10-17$. 